\title{
Novel daidzein analogs enhance osteogenic activity of bone marrow-derived mesenchymal stem cells and adipose-derived stromal/stem cells through estrogen receptor dependent and independent mechanisms
}

Amy L Strong ${ }^{1}$, Jason F Ohlstein ${ }^{1}$, Quan Jiang ${ }^{2}$, Qiang Zhang ${ }^{2}$, Shilong Zheng ${ }^{2}$, Stephen M Boue ${ }^{3}$, Steven Elliott ${ }^{4}$, Jeffrey M Gimble ${ }^{1}$, Matthew E Burow ${ }^{4}$, Guangdi Wang ${ }^{2}$ and Bruce A Bunnell ${ }^{1 *}$

\begin{abstract}
Introduction: Osteoporosis is a disease characterized by low bone mineral density (BMD) and increased risk of fractures. Studies have demonstrated the use of phytoestrogens, or plant-derived estrogens, such as genistein and daidzein, to effectively increase osteogenic activity of bone marrow-derived mesenchymal stem cells (BMSCs). Herein, the effects of daidzein analogs on the osteogenic differentiation efficiency of human BMSC and adipose-derived stromal/stem cells (ASC) were explored.

Methods: BMSCs and ASCs underwent osteogenic differentiation in the presence of vehicle, 17ß-estradiol (E2), phytoestrogens, or daidzein analogs. Cells were stained for alkaline phosphatase (ALP) enzymatic activity, calcium deposition by alizarin red s, and phosphate mineralization by silver nitrate. Gene expression analysis was conducted on cells treated with daidzein analogs.
\end{abstract}

Results: Cells treated with E2, daidzein, or genistein increased calcium deposition by 1.6-, 1.5-, and 1.4-fold, respectively, relative to vehicle-treated BMSCs and 1.6-, 1.7-, and 1.4-fold relative to vehicle-treated ASCs, respectively. BMSCs treated with daidzein analog 2c, 2g, and 21 demonstrated a 1.6-, 1.6-, and 1.9-fold increase in calcium deposition relative to vehicle-treated BMSCs, respectively, while ASCs treated with daidzein analog $2 \mathrm{c}$, $2 \mathrm{~g}$, or $2 \mathrm{l}$ demonstrated a 1.7-, 2.0-, and 2.2-fold increase in calcium deposition relative to vehicle-treated ASCs, respectively. Additional analysis with BMSCs and ASCs was conducted in the more efficient compounds: $2 \mathrm{~g}$ and $2 \mathrm{~L}$. ALP activity and phosphate mineralization was increased in 2g- and 2l-treated cells. The analysis of lineage specific gene expression demonstrated increased expression of key osteogenic genes (RUNX2, C-FOS, SPARC, DLX5, SPP1, COL1A1, IGF1, SOST, and DMP1) and earlier induction of these lineage specific genes, following treatment with $2 \mathrm{~g}$ or 21 , relative to vehicle-treated cells. Estrogen receptor (ER) inhibitor studies demonstrated that ER antagonist fulvestrant inhibited the osteogenic differentiation of $2 \mathrm{~g}$ in BMSCs and ASCs, while fulvestrant only attenuated the effects of 21 , suggesting that $2 \mathrm{l}$ acts by both ER dependent and independent pathways.

Conclusions: These studies provide support for exploring the therapeutic efficacy of daidzein derivatives for the treatment of osteoporosis. Furthermore, the patterns of gene induction differed following treatment with each daidzein analog, suggesting that these daidzein analogs activate distinct ER and non-ER pathways to induce differentiation in BMSCs and ASCs.

\footnotetext{
* Correspondence: bbunell@tulane.edu

${ }^{1}$ Center for Stem Cell Research and Regenerative Medicine, Tulane University School of Medicine, 1430 Tulane Avenue, SL-99, New Orleans, LA 70112, USA Full list of author information is available at the end of the article
} 


\section{Introduction}

Osteoporosis is a pathological condition associated with bone degeneration and is characterized by low bone mineral density (BMD) and alterations to the architecture of the bone. The low bone density and compromised architecture results in reduced bone strength and increased susceptibility to fractures, leading to significant morbidity and mortality [1-3]. While many factors contribute to the development of osteoporosis, age will probably be the leading risk factor due to the aging population in the United States [4]. It is estimated that more than 2 million people suffer from osteoporosis at a cost of $\$ 17$ billion annually in the United States [5]. Although increasing physical activity is a modifiable lifestyle choice that can reduce the incidence of osteoporosis [6], the development of novel therapeutic interventions will further reduce the development of osteoporosis by supporting healthier bones over an individual's lifetime.

Current treatment regimens for osteoporosis target bone regeneration or bone resorption, as these two processes are normally balanced in order to maintain strong, healthy bones. As such, therapeutic compounds have been divided into two groups: anti-resorptive drugs and anabolic drugs. Anti-resorptive drugs reduce the breakdown of bone during normal remodeling and reduce bone loss by limiting osteoclast activity [7]. These drugs include bisphosphonate, calcitonin, and denosumab. Studies have shown that delivery of these drugs independently or in combination is effective in reducing bone loss. While these drugs limit the severity of osteoporosis, it is still necessary for bone to undergo regeneration to restore the architecture of the bone and provide strength to the bones. Anabolic drugs have been shown not only to achieve higher BMD, but also to improve the quality and the strength of the bone [8].

Estrogens have anti-resorptive activity and anabolic activity, which have made them useful for the treatment of osteoporosis in postmenopausal women $[9,10]$. However, the precise mechanism by which this occurs remains to be determined. Furthermore, while estrogens are considered powerful modulators of bone metabolism by reducing the development of osteoporosis and increasing BMD, their use in the form of hormone replacement therapy has been halted due to its association with an increased risk of developing breast and endometrial cancer [11-13]. Effective alternatives to estrogens are therefore necessary. Raloxifene, a selective estrogen receptor (ER) modulator, has been shown to produce estrogenagonistic effects on bone and estrogen-antagonistic effects on uterine, endometrium, and breast tissue [14]. However, raloxifene has also been associated with increased risk of thromboembolic events [15]. There thus remains a need to identify superior pharmacological therapies to treat osteoporosis.
Plant-derived estrogens, or phytoestrogens, have gained significant attention and interest because these compounds have been shown to increase osteogenesis without increasing the risk of developing cancer [16-18]. More specifically, phytoestrogens isolated from soy, namely genistein and daidzein, are able to inhibit the bone resorption activity of osteoclasts while simultaneously stimulating osteogenic differentiation and maturation of bone marrow-derived mesenchymal stem cells (BMSCs) and osteoblasts [19]. In addition, previous studies have shown that these compounds have the ability to induce osteogenesis in osteoporosis-induced ovariectomized animal models [20].

While these studies are encouraging, the osteogenic potential of these phytoestrogens is less potent than the estrogens. Developing compounds with increased osteogenic potential without the tumorigenic potential of estrogens therefore remains a priority. Previously, our group designed and synthesized daidzein analogs that exhibited a wide range of estrogenic, anti-estrogenic, and osteogenic activity $[21,22]$. Herein, the impact of these daidzein analogs on the osteogenic potential of human BMSCs and adipose derived stromal/stem cells (ASCs) is described. Although previous studies have focused on BMSCs as the precursor to osteoblasts, recent studies have shown that ASCs differentiate into osteoblasts [23,24]. Additionally, the ease in isolation of ASCs and their abundance from adipose tissue makes them ideal candidates for tissue engineering and regenerative purposes.

To characterize the effect of these daidzein analogs (2c, 2g, and 2l) on BMSCs and ASCs, treated cells were assessed for the amount of calcium deposition by alizarin red staining, alkaline phosphatase (ALP) activity by 5-bromo-4-chloro-3-indolyl phosphate (BCIP/NBT) staining, and phosphate mineralization by silver nitrate staining. Furthermore, the dose of these compounds necessary to elicit $50 \%$ of the maximal osteogenic effect $\left(\mathrm{EC}_{50}\right)$ on BMSCs and ASCs was identified and their efficacy and potency was compared with $17 \beta$-estradiol (E2). To determine the downstream targets of these compounds, mRNA expression analysis was conducted on key osteogenic factors. Together, these studies provide support for daidzein analogs as novel therapeutic compounds that have the potential to reduce osteoporosis through increasing osteogenesis.

\section{Materials and Methods \\ Materials}

Anti-CD45-PeCy7, anti-CD11b-PeCy5, anti-CD166phycoerythrin, anti-CD105-phycoerythrin, anti-CD 90-PeCy5, anti-CD34-phycoerythrin, isotype control fluorescein isothiocyanate human $\mathrm{IgG}_{1}$, and isotypecontrol phycoerythrin human $\operatorname{IgG}_{2 a}$ were purchased from Beckman Coulter (Indianapolis, IN, USA). Anti- 
CD44-allophycocyanin was purchased from BD Biosciences (San Jose, CA, USA). Type 1 collagenase, bovine serum albumin (fraction V), calcium chloride, dexamethasone, isobuytlmethylxanthine, indomethacin, ascorbate 2 -phosphate, $\beta$-glycerol phosphate, alizarin red $\mathrm{S}$, oil red $\mathrm{O}$, cetylpyridinium chloride, $\mathrm{BCIP} / \mathrm{NBT}$, silver nitrate, E2, daidzein, and genistein were purchased from Sigma (St Louis, MO, USA). Daidzein analogs (i.e. 2c, 2g, and 2l) were synthesized in our laboratory as described previously $[21,22]$.

\section{Human subjects}

Primary human BMSCs were obtained from six healthy consenting Caucasian female donors under a protocol approved by Tulane University Institutional Review Board. The cells were prepared from bone marrow aspirates taken from the iliac crest of six individuals. Nucleated cells were isolated using Ficoll-Paque density gradient (Amersham Pharmacia Biotech, Milwaukee, WI, USA) and resuspended in complete culture media (CCM), which consisted of $\alpha$-Minimal Essential Medium (Gibco, Grand Island, NY, USA), 20\% fetal bovine serum (Atlanta Biologicals, Lawrenceville, GA, USA), 100 units/ml penicillin/ $100 \mu \mathrm{g} / \mathrm{ml}$ streptomycin (Gibco), and $2 \mathrm{mML}$-glutamine (Gibco). The cells were then seeded on a $150 \mathrm{~cm}^{2}$ culture dish (Nunc, Rochester, NY, USA) and maintained in a humidified $5 \%$ carbon dioxide $\left(\mathrm{CO}_{2}\right)$ incubator at $37^{\circ} \mathrm{C}$. Medium was changed every 3 to 4 day. When the cultures reached $70 \%$ confluence, the cells were harvested with $0.25 \%$ trypsin/1 mM ethylenediamine tetraacetic acid (EDTA; Gibco) and cryopreserved prior to experimental use.

Primary human ASCs were obtained from six healthy consenting Caucasian female donors undergoing elective liposuction procedures under a protocol approved by Pennington Biomedical Research Center Institutional Review Board. ASCs were isolated from processed lipoaspirates from the subcutaneous adipose tissue of subjects. Lipoaspirates were incubated in $0.1 \%$ type I collagenase and $1 \%$ bovine serum albumin dissolved in $100 \mathrm{ml}$ phosphate-buffered saline (PBS) supplemented with $2 \mathrm{mM}$ calcium chloride. The mixture was placed in a $37^{\circ} \mathrm{C}$ shaking water bath at $75 \mathrm{rpm}$ for 60 minutes and then centrifuged to remove oil, fat, primary adipocytes, and collagenase solution, leaving behind a pellet of cells. Cells were resuspended in CCM, plated on $150 \mathrm{~cm}^{2}$ culture dishes, and maintained in a humidified $5 \% \mathrm{CO}_{2}$ incubator. Fresh medium was added every 2 to 3 days until cells achieved 80 to $90 \%$ confluence and were harvested with $0.25 \%$ trypsin/ $1 \mathrm{mM}$ EDTA and cryopreserved prior to experimental use.

\section{Cell culture}

Frozen vials of approximately $10^{6}$ BMSCs or ASCs were thawed, plated onto $150 \mathrm{~cm}^{2}$ culture dishes (Nunc) in
$20 \mathrm{ml} \mathrm{CCM}$ and incubated at $37^{\circ} \mathrm{C}$ with $5 \%$ humidified $\mathrm{CO}_{2}$. After 24 hours, medium was removed and adherent, viable cells were washed with $\mathrm{PBS}$, harvested with $0.25 \%$ trypsin/1 mM EDTA (Gibco), and replated at 100 cells $/ \mathrm{cm}^{2}$ in CCM. Medium was replaced every 3 to 4 days. For all experiments, subconfluent cells $(<70 \%$ confluence) between passages 2 and 6 were used.

\section{Flow cytometry}

BMSCs and ASCs were harvested with $0.25 \%$ trypsin/ $1 \mathrm{mM}$ EDTA for 3 to 4 minutes at $37^{\circ} \mathrm{C}$. A total of $3 \times$ $10^{5}$ cells were suspended in $50 \mu \mathrm{l}$ PBS and incubated with fluorescence-labeled primary antibodies. The samples were incubated for 30 minutes at room temperature and washed with PBS. The samples were then analyzed with Galios Flow Cytometer (Beckman Coulter, Brea, CA, USA) running Kaluza software (Beckman Coulter). To assay cells by forward and side scatter, FACScan was standardized with microbeads (Dynosphere uniform microspheres; Bangs Laboratories Inc., Thermo Scientific, Waltham, MA, USA). At least 10,000 events were analyzed and compared with isotype controls.

\section{Colony-forming unit assay}

BMSCs and ASCs were plated at a density of 100 cells on a $10 \mathrm{~cm}^{2}$ plate in CCM and incubated for 14 days. Plates were then rinsed with PBS and stained with $3 \%$ crystal violet (Sigma) for 30 minutes at room temperature. Plates were washed with PBS and once with tap water. Colonies that were larger than $2 \mathrm{~mm}$ in diameter were counted.

\section{Differentiation protocols \\ Osteogenic differentiation}

BMSCs and ASCs were cultured in six-well plates in CCM until 70\% confluence. Medium was replaced with fresh osteogenic differentiation medium (ODM) consisting of $50 \mu \mathrm{M}$ ascorbate 2-phosphate, $10 \mathrm{mM} \beta$-glycerol phosphate, and $10 \mathrm{nM}$ dexamethasone. Where indicated, the fetal bovine serum in ODM was substituted for charcoal dextran-stripped fetal bovine serum (charcoal dextranstripped osteogenic differentiation medium (CDS-ODM); Atlanta Biologicals).

\section{Adipogenic differentiation}

BMSCs and ASCs were cultured in six-well plates in CCM until 70\% confluence. Medium was replaced with fresh adipogenic induction media made with CCM supplemented with $0.5 \mu \mathrm{M}$ dexamethasone, $0.5 \mathrm{mM}$ isobuytlmethylxanthine, and $50 \mu \mathrm{M}$ indomethacin. Where indicated, the fetal bovine serum in adipogenic differentiation medium was substituted for charcoal dextran-stripped adipogenic differentiation medium (CDS-ADM). 


\section{Phytoestrogen, daidzein analog, and fulvestrant treatment}

BMSCs and ASCs were plated in CCM and allowed to adhere to plastic. The medium was replaced with CDSODM or CDS-ADM and supplemented with vehicle, $10 \mathrm{nM}$ E2, $1 \mu \mathrm{M}$ daidzein, $1 \mu \mathrm{M}$ genistein, or $1 \mu \mathrm{M}$ daidzein analog 2c, 2g, or $2 \mathrm{l}$ for 14 days. Furthermore, where indicated, cells were treated with CDS-ODM and supplemented with log-fold increases of E2, daidzein, or daidzein analog from $100 \mathrm{pM}$ to $1 \mathrm{mM}$. ER antagonist studies were conducted by concurrent treatment with $100 \mathrm{nM}$ fulvestrant (ICI187,280; Sigma) and vehicle, $10 \mathrm{nM} \mathrm{E2,} 1 \mu \mathrm{M}$ daidzein, or $1 \mu \mathrm{M}$ daidzein analog $2 \mathrm{~g}$ or $2 \mathrm{l}$ in CDS-ODM.

\section{Staining and quantification protocols \\ Alizarin red staining and quantification}

After 14 days, cells undergoing osteogenic differentiation in ODM or CDS-ODM were fixed in 10\% formalin for 1 hour, washed with distilled water, and stained with $1 \%$ alizarin red ( $\mathrm{pH} 4.1)$ to visualize calcium deposition in the extracellular matrix as a marker of early osteogenesis. Images were acquired at $4 \times$ magnification on a Nikon Eclipse TE200 with a Nikon Digital Camera DXM1200F using Nikon ACT-1 software (Melville, NY, USA). For quantification, alizarin red was extracted from each well with $10 \%$ cetylpyridinium chloride and read at $584 \mathrm{~nm}$ (FLUOstar optima; BMG Labtech, Durham, NC ). To normalize to the amount of protein in each sample, protein extraction with RIPA buffer (Pierce, Thermo Scientific) and protein quantification with the BCA assay (Thermo Scientific) were performed according to manufacturer's instructions.

\section{Oil Red $O$ staining and quantification}

After 14 days, cells undergoing adipogenic differentiation in adipogenic differentiation medium or CDS-ADM were fixed in $10 \%$ formalin for 1 hour, and stained with oil red O, composed of two parts PBS and three parts $0.5 \%$ oil red $\mathrm{O}$ stock solution to visualize neutral lipids. Images were acquired at $10 \times$ magnification on a Nikon Eclipse TE200 with a Nikon Digital Camera DXM1200F using the Nikon ACT-1 software. For quantification, oil red O was extracted from each well with isopropanol and read at $544 \mathrm{~nm}$ (FLUOstar optima). To normalize to the amount of protein in each sample, protein extraction with RIPA buffer (Pierce, Thermo Scientific) and protein quantification with the BCA assay (Thermo Scientific) were performed according to manufacturer's instructions.

\section{$B C I P / N B T$ staining}

After 3 days, cells undergoing osteogenic differentiation in CDS-ODM were fixed in $10 \%$ formalin for 1 hour, washed with distilled water, and incubated in BCIP/NBT to visualize ALP activity. Images were acquired at $4 \times$ magnification on a Nikon Eclipse TE200 with a Nikon Digital Camera DXM1200F using Nikon ACT-1 software.

\section{Silver nitrate staining}

After 14 days, cells undergoing osteogenic differentiation in CDS-ODM were fixed in 10\% formalin for 1 hour, washed with distilled water, and incubated in 3\% silver nitrate to visualize phosphate mineralization in the extracellular matrix as a late marker of osteogenesis. Images were acquired at $4 \times$ magnification on a Nikon Eclipse TE200 with a Nikon Digital Camera DXM1200F using Nikon ACT-1 software.

\section{MTT assay}

Assessment of cell viability was performed with the MTT assay. BMSCs and ASCs were plated in triplicate in 96well plates (500 cells/well) in CCM supplemented with vehicle, daidzein analog $2 \mathrm{~g}$, or daidzein analog 2l. After 7 and 14 days, cells were incubated with $10 \mathrm{mM} \mathrm{MTT}$ (Invitrogen, Grand Island, NY, USA) for 4 hours at $37^{\circ} \mathrm{C}$ with $5 \%$ humidified $\mathrm{CO}_{2}$. A total of $100 \mu$ dissolving solution (10\% SDS, $0.01 \mathrm{M} \mathrm{HCl})$ was added to each well and incubated for 12 to 16 hours at $37^{\circ} \mathrm{C}$. Absorbance was measured at $544 \mathrm{~nm}$ (FLUOstar optima).

\section{RNA isolation, cDNA synthesis, quantitative reverse transcription polymerase chain reaction}

Cells treated with vehicle, $10 \mathrm{nM}$ E2, $1 \mu \mathrm{M}$ daidzein, or $1 \mu \mathrm{M}$ daidzein analog in CDS-ODM were collected on days 3, 7, and 14. Where indicated, BMSCs and ASCs treated with vehicle, E2, daidzein, or daidzein analog were simultaneously treated with $100 \mathrm{nM}$ fulvestrant and collected on days 3,7 , and 14 . Total cellular RNA was extracted from BMSCs and ASCs using the RNeasy Mini Kit (Qiagen, Valencia, CA, USA), purified with DNase I digestion (Invitrogen) according to the manufacturer's instructions, and reverse transcribed using the SuperScript VILO cDNA synthesis kit (Invitrogen). Quantitative real-time polymerase chain reaction was performed using the EXPRESS SYBR GreenER qPCR SuperMix Kit (Invitrogen) according to the manufacturer's instructions. The following forward and reverse primer sequences were used to detect changes in gene expression: runt-related transcription factor 2 (RUNX2), 5' -CTCACTACCACACCTACCTG-3' and $5^{\prime}$-TCAATATGGTCGCCAAACAGATTC-3'; FBJ murine osteosarcoma viral oncogene homolog (c-Fos), 5' -CCTGTCAAGAGCATCAGCAG-3' and 5'-GTCAG AGGAAGGCTCATTGC-5'; osteonectin (SPARC), $5^{\prime}$-TG TGGGAGCTAATCCTGTCC-3' and 5' ${ }^{\prime}$-TCAGGACGTT CTTGAGCCAGT-3'; distal-less homeobox 5 (DLX5), 5' -TGGCCCGAGTCTTCAGCTAC' and 5' -TGGTTGG TCGGTCTCTTTCT-3'; secreted phosphoprotein 1 (SPP1), 5'-GCTCTAGAATGAGAATTGCACTG-3' and 5'-TGT CGGTCCTGAGGTAACTG-3'; collagen type 1 alpha 
(COL1A1), 5' -CATGTTCAGCTTTGTGGACCTC-3' and 5'-AGGTGATTGGTGGGATGTCTT-3'; insulin-like growth factor 1 (IGF1), 5'-CTGTGATCTAAGGAGGCTG-3'

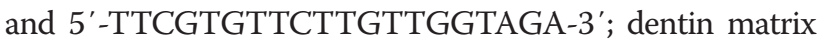
acidic phosphoprotein 1 (DMP1), 5'-GTGAGTGAGTC CAGGGGAGATAA-3' and 5' ${ }^{\prime}$-TTTTGAGTGGGAGAG TGTGTGC-3'; sclerostin (SOST), 5'-TCCCCACCACC CCTTTG-3' and 5' -GGTCACGTAGCGGGTGAA-3'; and $\beta$-actin, $5^{\prime}$-CACCTTCTACAATGAGCTGC-3' and 3'-TCTTCTCGATGCTCGACGGA-3'. All reverse transcription-polymerase chain reaction primers were designed using Primer3 (Free Software Foundation Inc.,Boston, MA, USA) and purchased from Integrated DNA Technologies (Coralville, IA, USA). The expression of human $\beta$-actin was used to normalize the mRNA content. Samples were tested in triplicate. No-template controls and no-reverse transcription controls were included in each polymerase chain reaction run.

\section{Statistical analysis}

All values are presented as the mean \pm standard deviation. Experiments were conducted separately with each donor, plated in triplicates for technical duplicates. The values for the technical duplicates for each donor were averaged together. The values for the six donors were averaged together to calculate the mean \pm standard deviation. The statistical differences between two or more groups were determined by analysis of variance, followed by post hoc Bonferroni multiple comparison tests. Statistical significant was set at $P<0.05$. Only statistically significant values were considered relevant. Analysis was performed using Prism (Graphpad Software, San Diego, CA, USA).

\section{Results}

BMSCs and ASCs demonstrate similar stem cell characteristics

BMSCs and ASCs were stained for cell surface antigens, plated for colony-forming units, and induced to differentiate down osteogenic and adipogenic lineages. BMSCs and ASCs displayed overlapping cell surface marker profiles $\left(\mathrm{CD} 44^{+}, \mathrm{CD}^{\circ} 0^{+}, \mathrm{CD} 105^{+}, \mathrm{CD}_{166}{ }^{+}, \mathrm{CD}_{11 b^{-}}, \mathrm{CD} 34^{-}\right.$, and $\mathrm{CD} 45^{-}$), were able to form colony-forming units, and underwent osteogenic and adipogenic differentiation (Additional file 1).

\section{Phytoestrogens enhance osteogenic differentiation while only estradiol enhanced adipogenic differentiation of BMSCs and ASCs}

BMSCs and ASCs were cultured in CDS-ODM and supplemented with vehicle, E2, daidzein, or genistein. After 14 days, cells were stained with alizarin red and imaged with bright-field microscopy (Figure 1A). Alizarin red staining was quantified by eluting the alizarin red staining and acquiring optical density measurements. E2-treated, daidzein-treated, and genistein-treated BMSCs demonstrated 1.6-fold, 1.5-fold, and 1.4-fold greater osteogenic differentiation compared with vehicle-treated BMSCs, respectively $(P<0.01$; Figure $1 B)$. ASCs treated with E2, daidzein, and genistein demonstrated a 1.6-fold, 1.7-fold, and 1.4-fold increase in osteogenic differentiation relative to vehicle-treated ASCs (normalized to 1.0), respectively $(P<0.01$; Figure $1 \mathrm{~B})$. It should be noted that while E2, daidzein, and genistein all enhanced osteogenic differentiation on average, two of the six BMSC donors and three of the six ASC donors did not respond to genistein treatment. Furthermore, visualization with bright-field microscopy did not demonstrate enhanced cell death in genistein-treated BMSCs or ASCs, compared with vehicle-treated cells.

To determine the effect of E2 or phytoestrogens on adipogenic differentiation of BMSCs and ASCs, cells were cultured in CDS-ADM and supplemented with E2, daidzein, and genistein. After 14 days, cells were stained with oil red $\mathrm{O}$ and imaged with bright-field microscopy (Figure 1C). Oil red staining was quantified by eluting with isopropanol and measuring the optical density of the extracted product. E2-treated BMSCs and ASCs demonstrated enhanced adipogenic differentiation by 1.6 -fold compared with vehicle-treated BMSCs and ASCs $(P<0.01$; Figure 1D). Daidzein enhanced adipogenic differentiation of BMSCs by 1.2-fold compared with vehicle-treated BMSCs $(P<0.01$; Figure 1D) but had no effect on the adipogenic differentiation of ASCs. Genistein also had no effect on the adipogenic differentiation of BMSCs or ASCs.

\section{Daidzein analogs enhance osteogenic differentiation while reducing adipogenic differentiation of BMSCs and ASCs}

To determine the osteogenic potential of daidzein analogs on BMSCs and ASCs, daidzein analogs 2c, 2g, and $2 \mathrm{l}$ were supplemented into CDS-ODM and at each media change. Cells were then stained with alizarin red and imaged with bright-field microscopy (Figure 1E), and the amount of staining was quantified by eluting with cetylpyridinium chloride. BMSCs treated with ana$\operatorname{logs} 2 \mathrm{c}, 2 \mathrm{~g}$, and $2 \mathrm{l}$ demonstrated a 1.6-fold, 1.6-fold, and 1.9-fold increase in osteogenesis, respectively, relative to vehicle-treated BMSCs $(P<0.05$; Figure 1F). ASCs treated with analogs $2 \mathrm{c}, 2 \mathrm{~g}$, and $2 \mathrm{l}$ demonstrated a similar trend and osteogenesis was enhanced by a 1.7-fold, 2.0-fold, and 2.2-fold increase, respectively, relative to vehicle-treated ASCs $(P<0.05$; Figure $1 \mathrm{~F})$. Furthermore, analog 2l-treated BMSCs and ASCs demonstrated enhanced osteogenesis compared with E2-treated and daidzein-treated BMSCs and ASCs $(P<0.001$; Figure 1F), suggesting that analog $2 \mathrm{l}$ is a more potent osteogenic compound than E2 or daidzein. 


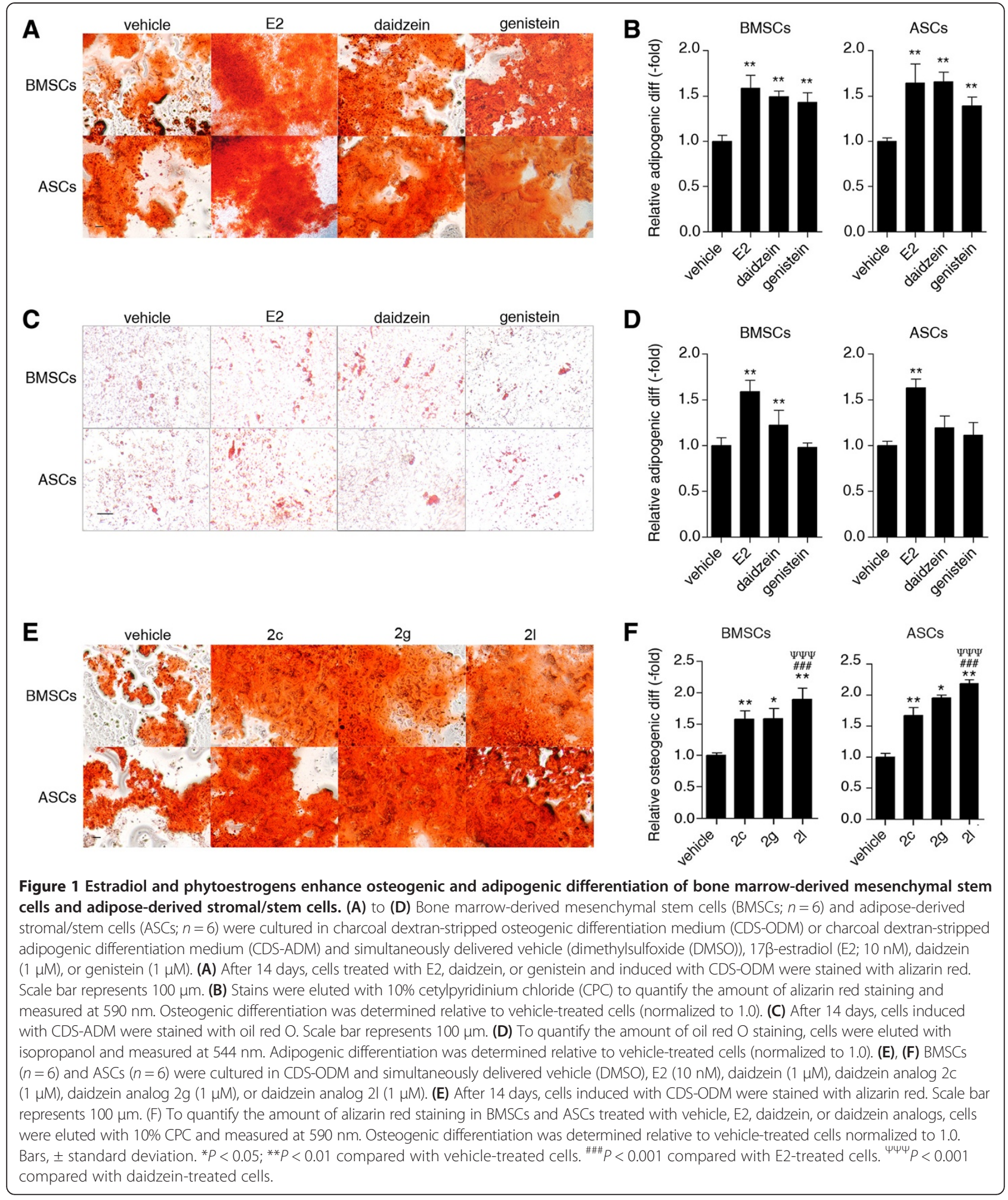

While analog 2c-treated, 2g-treated, and 2l-treated BMSCs and ASCs demonstrated enhanced osteogenic differentiation, these compounds failed to simulate adipogenic differentiation. BMSCs and ASCs treated with analog $2 c$ demonstrated adipogenic differentiation comparable with vehicle-treated BMSCs and ASCs, while analog 2g-treated and 2l-treated BMSCs and ASCs demonstrated a significant reduction in adipogenic differentiation compared with vehicle-treated, E2-treated, or daidzein-treated BMSCs or ASCs $(P<0.001$; Additional file 2$)$. 
To assess whether changes observed with differentiation were associated with changes caused by cytotoxicity or increased proliferation, BMSCs and ASCs were cultured in CCM supplemented with vehicle, daidzein analog $2 \mathrm{~g}$, or daidzein analog $2 \mathrm{l}$ and assessed after 7 and 14 days. Analog 2g-treated and 2l-treated BMSCs demonstrated similar growth rates to vehicle-treated BMSCs ( $P>0.05$; Additional file 3$)$. Likewise, 2g-treated and 2ltreated ASCs demonstrated similar rates of proliferation compared with vehicle-treated ASCs, as no difference was observed in cell number after 7 or 14 days $(P>0.05$; Additional file 3).

\section{Daidzein analogs $2 \mathrm{~g}$ and $2 \mathrm{l}$ have similar $\mathrm{EC}_{50}$ values but differ in effectiveness}

Additional studies were conducted to determine the concentration of daidzein analogs required to induce $\mathrm{EC}_{50}$ on osteogenesis of BMSCs and ASCs in the most potent compounds: $2 \mathrm{~g}$ and $2 \mathrm{l}$. Analogs $2 \mathrm{~g}$ and $2 \mathrm{l}$ were thus administered to BMSCs and ASCs at concentrations ranging from $100 \mathrm{pM}$ to $1 \mathrm{mM}$, at log-fold increases. The $\mathrm{EC}_{50}$ value for E2 in BMSCs and ASCs was $10^{-8.35}$ and $10^{-9.20}$, respectively (Additional file 4 ). The $\mathrm{EC}_{50}$ value for daidzein, analog $2 \mathrm{~g}$, and analog $2 \mathrm{l}$ in BMSCs was $10^{-6.62}, 10^{-6.99}$, and $10^{-6.99}$, respectively (Additional file 4 ). The $\mathrm{EC}_{50}$ values for daidzein, analog $2 \mathrm{~g}$, and analog $2 \mathrm{l}$ in ASCs were comparable with those for BMSCs: $10^{-7.76}, 10^{-7.53}$, and $10^{-7.32}$, respectively (Additional file 4). E2 displays the most potent stimulation of osteogenesis in BMSCs and ASCs, leading to enhanced osteogenesis at $1 \mathrm{nM}$ to $10 \mathrm{nM}$ concentrations. However, analog 21 treatment at $1 \mu \mathrm{M}$ concentration resulted in the greatest degree of osteogenic differentiation in BMSCs (2.0-fold) and ASCs (2.4-fold; Figure 2). Higher doses of E2 were unable to induce osteogenesis equal to or exceeding that of analog $2 \mathrm{l}(P<0.05$; Figure 2$)$.

\section{Enhanced alkaline phosphatase activity and increased phosphate deposition was observed in analog $2 \mathrm{~g}$-treated and 2l-treated BMSCs and ASCs}

Due to the enhanced efficacy of analogs $2 \mathrm{~g}$ and $2 \mathrm{l}$, additional techniques were utilized to determine whether cells treated with $2 \mathrm{~g}$ or $2 \mathrm{l}$ enhanced early osteogenesis. BMSCs and ASCs treated with vehicle, E2, daidzein, analog $2 \mathrm{~g}$, or analog 21 for 3 days were incubated in BCIP/NBT, the substrate for ALP. E2 enhanced BMSC and ASC differentiation by 2.0 -fold and 1.6-fold, respectively; analog $2 \mathrm{~g}$ enhanced differentiation by 2.1-fold and 2.4-fold; and ana$\log 21$ enhanced differentiation by 1.9 -fold and 2.6 -fold $(P<0.05$; Figure 3A,B). While daidzein-treated BMSCs demonstrated enhanced ALP activity (1.6-fold increase; $P<0.05)$ relative to vehicle, daidzein-treated ASCs displayed similar ALP activity to vehicle-treated ASCs.

To assess phosphate deposition, which is secreted during late osteogenesis and is an essential component of the mature extracellular matrix of bone, cells were treated with vehicle, E2, daidzein, analog $2 \mathrm{~g}$, or analog $2 \mathrm{l}$ for 14 days and incubated in silver nitrate. While E2treated and daidzein-treated BMSCs and ASCs demonstrated enhanced phosphate deposition, E2 treatment resulted in greater phosphate deposition than daidzein treatment $(P<0.001$; Figure $3 C, D)$. Structural modifications of daidzein into analogs $2 \mathrm{~g}$ and $2 \mathrm{l}$ resulted in increased phosphate deposition $(P<0.001$; Figure $3 C, D)$. Treatment of BMSCs and ASCs with analog 21 resulted in the most significant increase in phosphate deposition compared with all other treatment groups $(P<0.001$; Figure 3C,D).
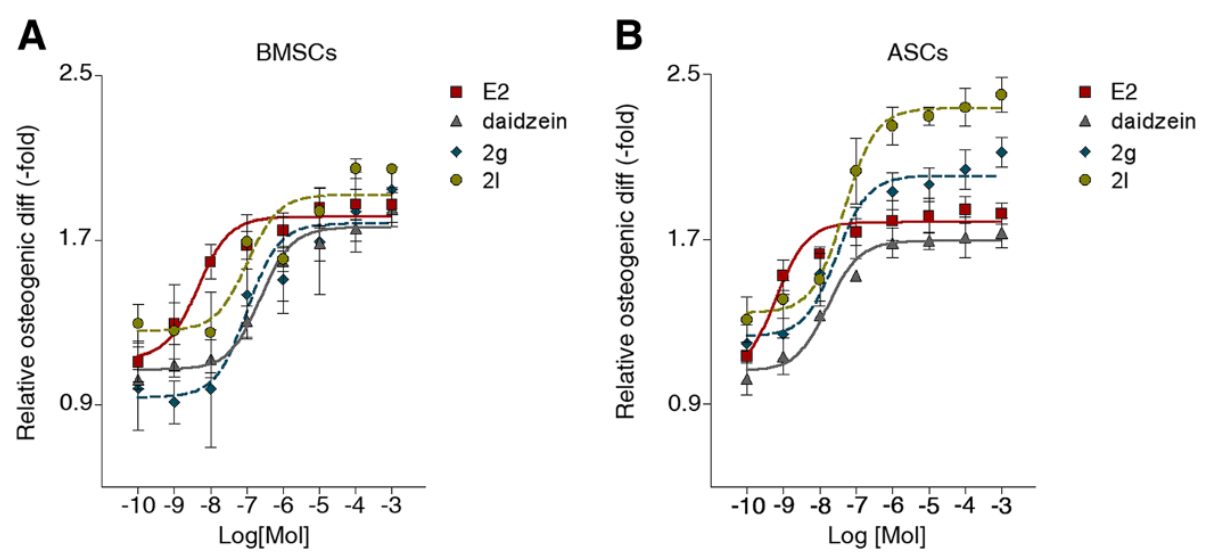

Figure 2 Effect of daidzein analogs on osteogenic differentiation of bone marrow-derived mesenchymal stem cells and adipose-derived stromal/stem cells is dose dependent. (A) Bone marrow-derived mesenchymal stem cells (BMSCs; $n=6)$ and (B) adipose-derived stromal/stem cells (ASCs; $n=6$ ) were cultured in charcoal dextran-stripped osteogenic differentiation medium and simultaneously treated with vehicle, $17 \beta$-estradiol (E2), daidzein, or daidzein analog $2 \mathrm{~g}$ or $2 \mathrm{l}$ at concentrations between $100 \mathrm{pm}$ and $1 \mathrm{mM}$. After 14 days, cells were fixed, stained with alizarin red, destained with cetylpyridinium chloride, and measured at $590 \mathrm{~nm}$. Osteogenic differentiation was determined relative to vehicle-treated cells normalized to 1.0. Bars, \pm standard deviation. 


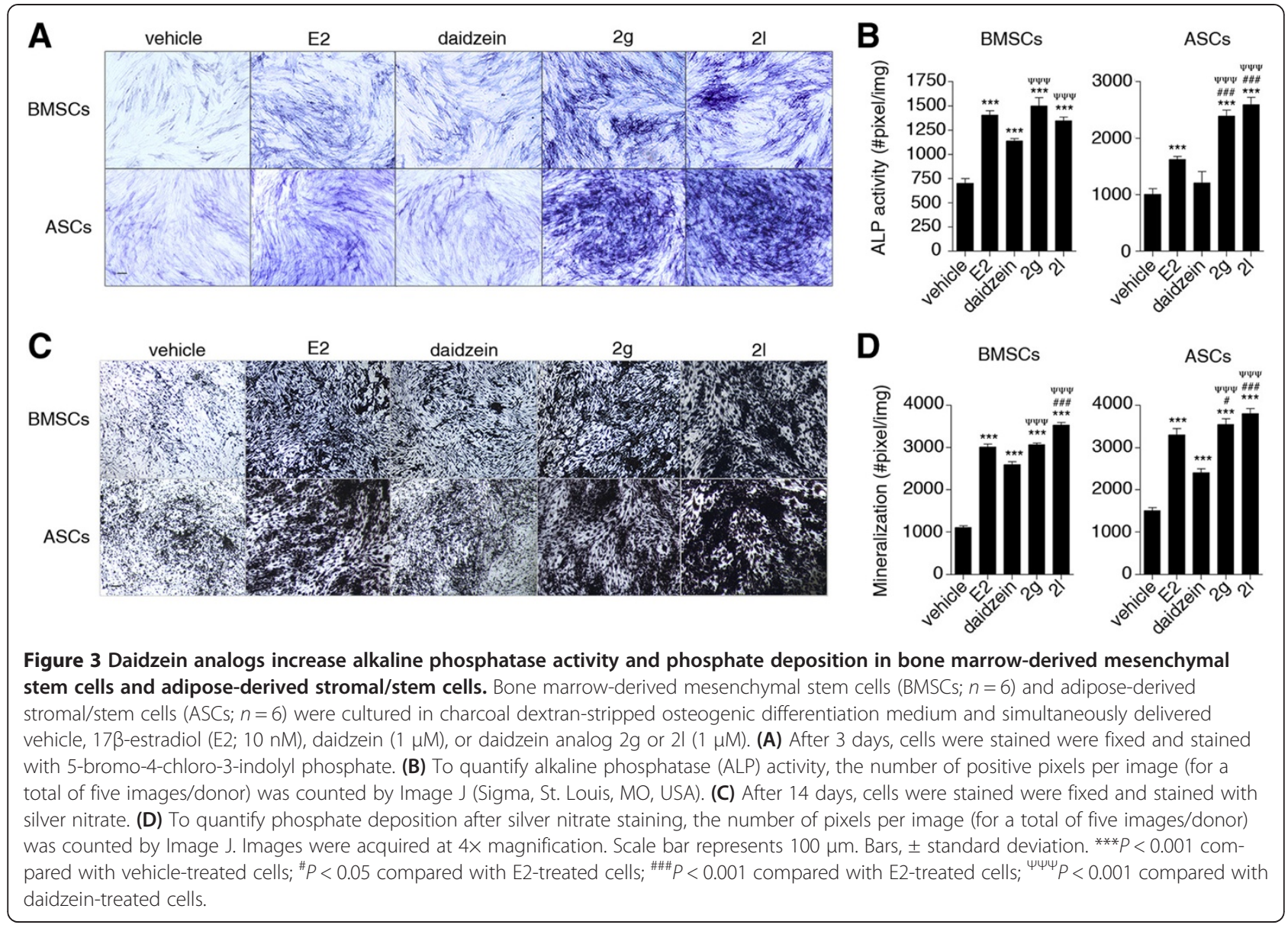

Together, these results suggest that BMSCs and ASCs treated with E2 demonstrate enhanced osteogenesis through increased ALP activity and phosphate deposition. Daidzein analogs $2 g$ and 21 also resulted in enhanced ALP activity and phosphate deposition. Treatment with daidzein was less effective than treatment with E2 or daidzein analogs.

\section{BMSCs and ASCs treated with 17 $\beta$-estradiol demonstrate induction of early, middle, and late genes involved in osteogenesis}

While BMSCs and ASCs treated with E2 displayed similar temporal induction of early and late osteogenic genes, significant differences were observed in the induction of middle osteogenic genes (Figures 4 and 5). More specifically, E2 induced the expression of early osteogenic genes (c-FOS and COL1A1) within 3 days in both BMSCs and ASCs $(P<0.05$; Figures 4 and 5$)$. However, the levels of gene induction varied between BMSCs and ASCs. Following 3 days of treatment, E2 increased cFOS expression by 2.4-fold in BMSCs, while E2 enhanced c-FOS expression by 141.1-fold in ASCs, relative to undifferentiated cells. The induction of COL1A1 expression was different between BMSCs and ASCs: 3.4- fold in BMSCs and 18.3-fold in ASCs, relative to undifferentiated cells $(P<0.05$; Figures 4 and 5$)$. These results highlight the differences in response to E2 stimulation.

Furthermore, differences were observed in the effects of E2 on middle osteogenic genes both temporally and in relation to induction level. SPARC and DLX5 was upregulated following 7 days of E2 treatment in BMSCs, while SPARC, DLX5, and SPP1 was upregulated following 14 days of treatment in ASCs, relative to undifferentiated cells $(P<0.05$; Figures 4 and 5$)$. E2-treated BMSCs displayed higher levels of induction in the middle genes on day 7, relative to undifferentiated cells (SPARC, 6.9fold in BMSCs vs. 1.3-fold in ASCs; DLX5, 17.8-fold in BMSCs vs. 5.8-fold in ASCs; Figures 4 and 5).

Additional differences between BMSCs and ASCs associated with E2 stimulation were in RUNX2 and IGF1 expression. RUNX2 expression in BMSCs was increased (9.1-fold) following 7 days of E2 treatment, while enhanced RUNX2 expression in ASCs (22.0-fold) did not occur until 14 days, relative to undifferentiated cells $(P<0.05$; Figures 4 and 5$)$. IGF1 induction was significantly enhanced in BMSCs following E2 treatment after 3 days in BMSCs, while this effect was not observed in ASCs (Figures 4 and 5; Additional file 5). Together, these 

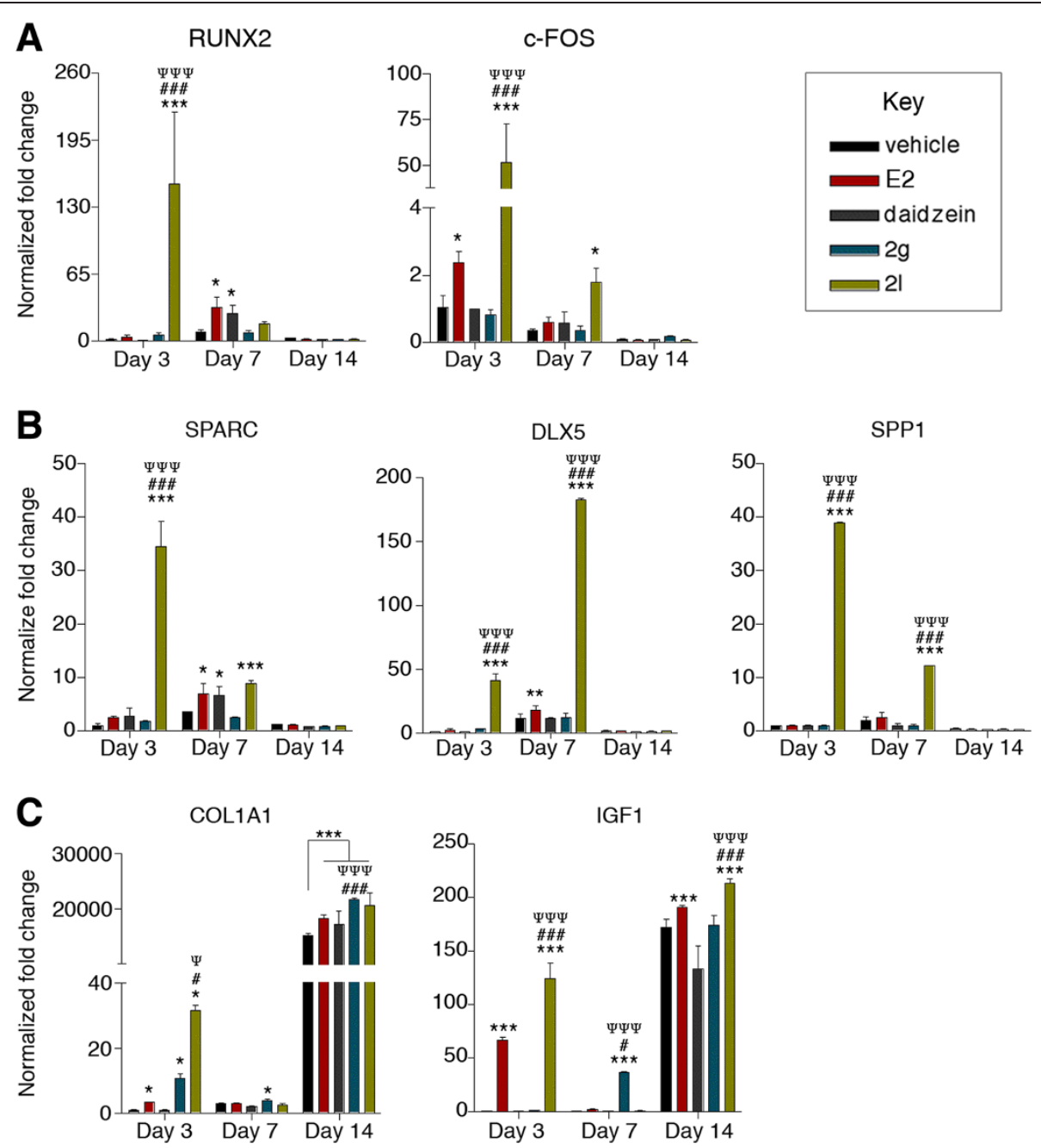

Figure 4 Expression of early, middle, and late-stage lineage-specific osteogenic genes in bone marrow-derived mesenchymal stem cells was significantly induced by daidzein analogs. Bone marrow-derived mesenchymal stem cells (BMSCs) were cultured in charcoal

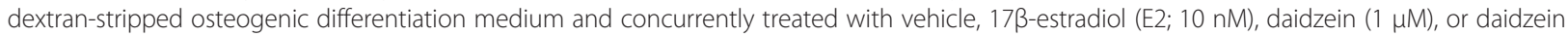
analog $2 \mathrm{~g}$ or $2 \mathrm{l}(1 \mu \mathrm{M})$. Cells were collected after 3,7 , or 14 days of treatment. RNA was isolated from the cells and reverse transcribed into cDNA. Analysis of (A) early, (B) middle, or (C) late osteogenic transcription factors by quantitative polymerase chain reaction. Expression values are normalized to undifferentiated cells normalized to 1.0. Bars, \pm standard deviation. ${ }^{*} P<0.05$ compared to vehicle-treated BMSCs; ${ }^{* *} P<0.001$ compared with vehicle-treated BMSCs; ${ }^{\#} P<0.05$ compared with E2-treated BMSCs; ${ }^{\# \# \#} P<0.001$ compared with E2-treated BMSCs; ${ }^{\psi} P<0.05$ compared with daidzein-treated BMSCs; ${ }^{\psi \psi \psi} P<0.001$ compared with daidzein-treated BMSCs.

results suggest that E2 stimulates the expression of osteogenic genes in both BMSCs and ASCs.

\section{Daidzein-treated, analog 2g-treated, and analog 2l-treated BMSCs and ASCs display different gene expression profiles}

BMSCs and ASCs were treated with daidzein and collected after 3, 7, and 14 days. The expression of key osteogenic factors was investigated by quantitative polymerase chain reaction. Daidzein-treated BMSCs increased RUNX2 and SPARC expression by 9.1-fold and 3.6-fold, respectively, relative to undifferentiated BMSCs $(P<0.05$;
Figure 4). Daidzein-treated ASCs demonstrated increased c-FOS expression by 134.9-fold after 3 days, and COL1A1 and IGF1 expression by 3,899.6-fold and 10.2-fold, respectively, after 14 days, compared with undifferentiated ASCs $(P<0.001$; Figure 5$)$.

In contrast, BMSCs treated with analog $2 \mathrm{~g}$ increased RUNX2 expression by 7.9-fold on day 7 and COL1A1 on days 3,7 , and 14 by 10.8 -fold, 4.1-fold, and 21,600.4-fold, respectively $(P<0.05$; Figure 4$)$. The expression of COL1A1 was significantly more robust compared with E2-treated or daidzein-treated BMSCs on day 14 ( $P<0.001$; Figure $4 C)$. ASCs treated with analog $2 \mathrm{~g}$ demonstrated increased expression of COL1A1 compared with vehicle-treated, 


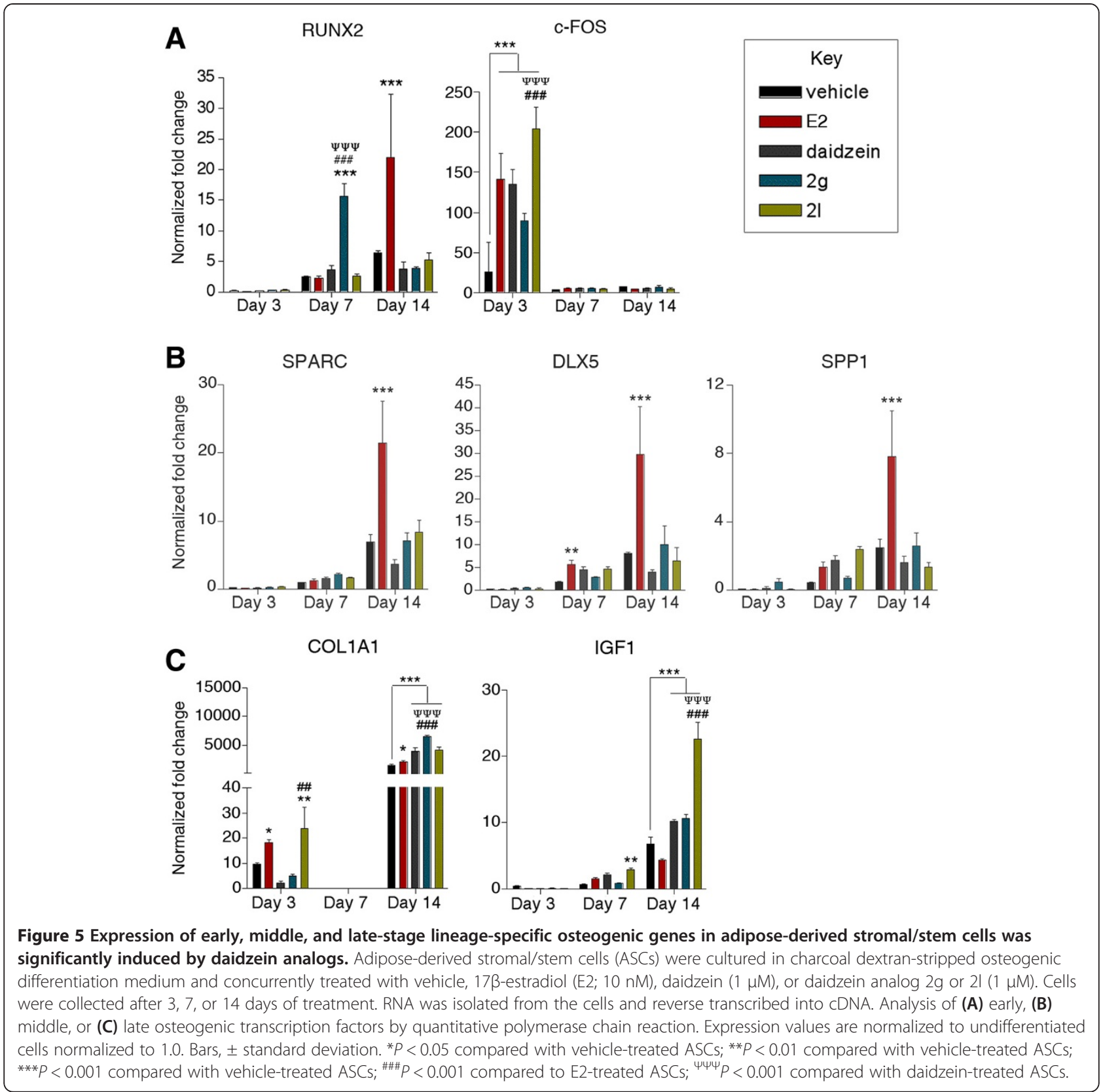

E2-treated, or daidzein-treated ASCs on day $14(P<0.001$; Figure 5C).

Unlike E2, daidzein, or analog 2g, BMSCs treated with analog 21 showed increased expression of all early, middle, and late osteogenic genes investigated in this study. Relative to undifferentiated BMSCs, BMSCs treated with ana$\log 2 \mathrm{l}$ demonstrated an enhanced expression of RUNX2, c-FOS, SPARC, DLX5, SPP1, COL1A1, and IGF1 by 152.8-fold, 51.6-fold, 34.4-fold, 41.2-fold, 38.8-fold, 31.8 -fold, and 124.4-fold, respectively, on day $3(P<0.05$; Figure 4). Daidzein analog 21 continued to induce the expression of c-FOS, SPARC, DLX5, and SPP1 by 1.8-fold, 8.9-fold, 182.8-fold, and 12.3-fold, respectively, on day 7
$(P<0.05$; Figure 4). Additionally, analog 21-treated BMSCs displayed increased late osteogenic genes COL1A1 and IGF1 even on day 14 $(P<0.05$; Figure 4$)$. These results suggest that analog $2 \mathrm{l}$ significantly induces osteogenesis through induction of key osteogenic regulatory genes to initiate and maintain osteogenesis.

While the effects of analog 21 on BMSCs were pronounced, the effects of analog $2 \mathrm{l}$ on ASCs were attenuated. ASCs treated with analog 21 displayed enhanced c-FOS expression by 204.5 -fold after 3 days $(P<0.05$; Figure 5). After 14 days of differentiation, analog 2ltreated ASCs displayed higher expression of COL1A1 (4,139.8-fold) compared with vehicle-treated (1,471.7-fold) 
or E2-treated (2,061.2-fold) ASCs $(P<0.05$; Figure 5). Likewise, analog 2l-treated ASCs demonstrated enhanced IGF-1expression (22.6-fold) after 14 days, compared with vehicle-treated ASCs (6.8-fold) or E2-treated ASCs (4.4fold; $P<0.05$; Figure 5).

\section{Fulvestrant reversed the osteogenic effects of daidzein and analog $2 \mathrm{~g}$ while only attenuating the effects of analog 21}

To determine the estrogenic activity of daidzein, ER inhibitor studies were performed on BMSCs and ASCs by treating cells simultaneously with daidzein and fulvestrant. Fulvestrant reduced the osteogenic potential of daidzein-treated BMSCs and ASCs from 1.7-fold to 1.0fold and from 1.5-fold to 1.0-fold, respectively (Figure 6). While daidzein increased the expression of RUNX2 on day 7 , simultaneous treatment with fulvestrant reduced
RUNX2 expression by $56.0 \%$, from 3.0 -fold to 1.3 -fold $(P<0.05$; Figures 4 and 6; Additional files 6 and 7$)$. With respect to ASCs, concomitant fulvestrant treatment reduced daidzein-induced c-FOS expression by $66.7 \%$ on day 3 (from 1.5 -fold to 0.5 -fold; $P<0.001$ ), IGF1 by $85.7 \%$ on day 7 (from 2.8 -fold to 0.4 -fold; $P<0.01$ ), and COL1A1 by $42.3 \%$ on day 14 (from 2.6 -fold to 1.5 -fold; $P<0.05$; Figures 4 and 6; Additional files 5, 7 and 8). These inhibitor studies suggest that daidzein works through an ER-dependent mechanism as fulvestrant treatment reduced expression of genes activated by daidzein.

To investigate the activation of ER signaling pathways by analog $2 \mathrm{~g}$, inhibitor studies were conducted with BMSCs and ASCs by simultaneously treating cells with analog $2 \mathrm{~g}$ and fulvestrant in CDS-ODM for 3,7 , or 14 days. Fulvestrant treatment reduced the osteogenic activity of analog $2 \mathrm{~g}$ in BMSCs and ASCs to levels

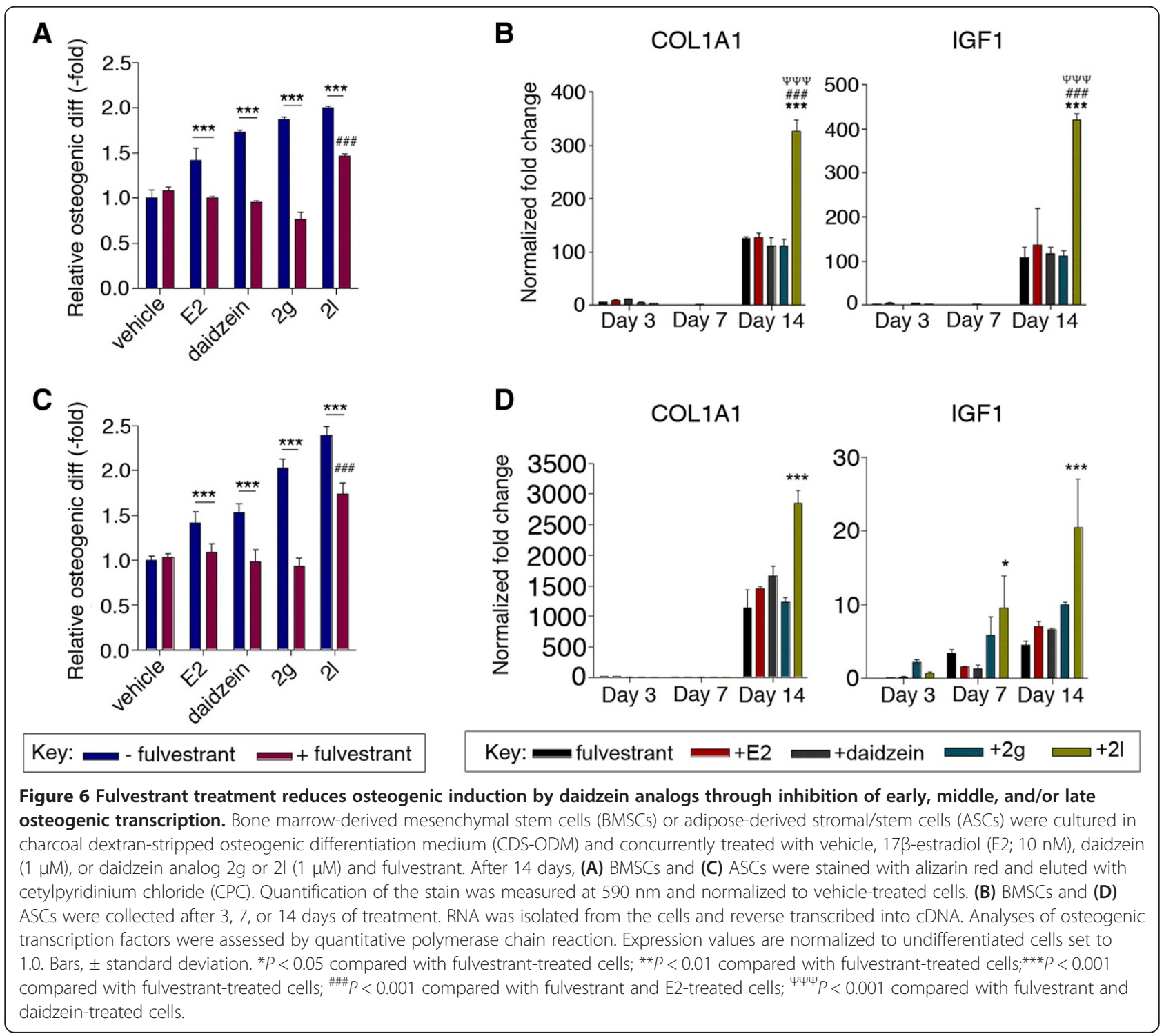


comparable with fulvestrant-treated cells from 1.9-fold to 0.8 -fold and from 2.0-fold to 0.9 -fold, respectively $(P<0.001$; Figure 6). Furthermore, BMSCs treated with analog $2 \mathrm{~g}$ and fulvestrant displayed reduced RUNX2 expression by $79.0 \%$ (from 4.3 -fold to 0.9 -fold) on day 3, COL1A1 expression by $91.6 \%$ (from 10.8-fold to 0.9 -fold) on day 3, and IGF1 expression by $97.8 \%$ (from 40.9 -fold to 0.9 -fold) on day 7 ( $P<0.05$; Additional files 5, 6 and 7). With respect to ASCs, fulvestrant inhibited the activity of analog $2 \mathrm{~g}$ by reducing RUNX2 expression on day 7 by 65.1\% (from 6.3-fold to 2.2-fold) and COL1A1 expression on day 14 by $75 \%$ (from 4.4 -fold to 1.1 -fold; $P<0.01$; Additional files 7, 8 and 9). Together, these results indicate that analog $2 \mathrm{~g}$ acts through an ER-mediated mechanism, with reduced activity in the presence of fulvestrant.

Furthermore, to determine the estrogenic activity of analog 2l, concomitant treatment of $2 \mathrm{l}$ with fulvestrant resulted in reduced osteogenesis in BMSCs and ASCs. Osteogenic differentiation was not completely inhibited by fulvestrant, suggesting that analog 2l-induced osteogenic differentiation is through both ER-dependent and ER-independent pathways. Simultaneous delivery of fulvestrant with analog $2 \mathrm{l}$ reduced the osteogenesis of BMSCs (2.0-fold to 1.5-fold) and ASCs (2.4-fold to 1.7-fold; $P<0.001$; Figure 6). Furthermore, fulvestrant reduced the expression of early and middle osteogenic genes in both BMSCs and ASCs, suggesting that the estrogenic mechanism of fulvestrant on osteogenesis is through the induction of early to middle osteogenic genes. COL1A1 and IGF1 expression remained induced in BMSCs even in the presence of fulvestrant (Figure 6; Additional files 2 and 9), suggesting that analog 21 utilizes an alternative non-ERdriven pathway to induce osteogenesis. Lastly, due to the impact of analog $2 \mathrm{l}$ on late osteogenic genes COL1A1 and IGF1, analysis of additional late osteogenic genes was conducted. Treatment with analog $2 \mathrm{l}$ increased DMP1 (3.9fold) and SOST (2.2-fold) expression in BMSCs, while 21 had no effect on DMP1 or SOST expression in ASCs (Additional file 10). In BMSCs, fulvestrant treatment reversed the increased expression in SOST by analog 21 but did not alter DMP1 expression (Additional file 10). These data suggest that analog 21 selectively induces osteogenesis through non-ER-dependent mechanisms that result in the upregulation of COL1A1, IGF1, and SOST in BMSCs and of COLA1A and IGF1 in ASCs.

\section{Discussion}

Osteoporosis is a disease characterized by decreasing BMD and a loss of the bone architecture, resulting in increased fragility and fracture incidence. To reduce the progression of the disease and increase bone strength, the development of new compounds to increase osteogenesis is necessary. In this study, synthetic daidzein analogs have been tested for their in vitro osteogenic potential in both BMSCs and ASCs. Daidzein analogs $2 \mathrm{~g}$ and $2 \mathrm{l}$ were found to increase osteogenic differentiation characterized by alizarin red staining, ALP activity, and silver nitrate staining. In addition, these daidzein analogs enhanced osteogenic differentiation of BMSCs and ASCs relative to E2-treated or daidzein-treated cells. Furthermore, simultaneous treatment with fulvestrant eliminated the osteogenic activity of daidzein analog $2 \mathrm{~g}$ and attenuated the osteogenic activity of analog $2 \mathrm{l}$, suggesting that $2 \mathrm{~g}$ acts predominantly through ER signaling while 21 may use both ER-dependent and ER-independent pathways. Analysis of transcript levels of key osteogenic genes showed that analogs $2 \mathrm{~g}$ and $2 \mathrm{l}$ differentially induced osteogenic genes in BMSCs and ASCs. These results raise the potential that individual daidzein analogs may function through distinct ER signaling mechanisms such as ER $\alpha, E R \beta$ or the G-protein-coupled ER. Recent research has demonstrated the involvement of G-protein-coupled ER as a mechanism of rapid ER signaling that can cross-talk with classic ER mechanisms or function in a distinct manner [25-27]. A combination of $E R \alpha / \beta$-mediated and G-protein-coupled ER-mediated mechanisms may thus exist by which daidzein analogs influence the MSC and ASC differentiation responses. Evidence has also demonstrated that fulvestrant alone exhibits effects on gene expression apart from its anti-estrogenic effects, which further supports the possibility that certain daidzein analogs may function through distinct G-protein-coupled ER-dependent or ER-independent pathways [28-30].

Consistent with previously published studies, genistein and daidzein increased the osteogenic potential of BMSCs and ASCs. Previous work by Bitto and colleagues demonstrated that genistein enhanced the BMD but also restored structure to ovariectomy-induced osteoporotic bone in rats [31,32]. Furthermore, the effects of genistein treatment in rats improved the overall architecture and strength of the bone better than raloxifene, a commonly used selective ER modulator used to treat osteoporosis $[31,32]$. Comparative studies have shown that daidzein is more effective than genistein in preventing ovariectomyinduced bone loss in rats [33]. Indeed, daidzein was shown to enhance BMD in lumbar vertebrae, femur, and in the metaphyseal and diaphyseal zones, which have been shown to be rich in cancellous and cortical bone, respectively [33]. Daidzein treatment has also been shown to increase biomechanical strength by increasing collagen formation, while reducing osteoclast activity to limit the amount of degradation to the extracellular matrix $[34,35]$. Together, daidzein treatment leads to reduced resorptive activity and increased anabolic activity in bone. The results of this study provide additional support for the anabolic activity of daidzein in BMSCs and ASCs. Additional studies have shown that daidzein with high calcium 
preserves bone mass and biomechanical strength in multiple sites in an ovariectomized mouse model [36], providing for the supplementation of daidzein with current osteoporosis treatment regimes.

While these phytoestrogens have proven effective in increasing bone density in rodent models, novel daidzein derivatives developed by our group were tested on BMSCs and ASCs to determine their potential to enhance bone differentiation and regeneration. Studies have shown that derivatives of genistein and daidzein have yielded better outcomes as anti-osteoporotic compounds compared with their original forms, either increasing anabolic activity or decreasing resorption activity. Wang and colleagues demonstrated that genistein derivatives act as potential selective ER modulators and increased the weight of bone in the femur relative to no treatment or treatment with genistein [37]. Other soy derivatives have been shown to increase in vitro osteoblast maturation in primary cultures of rat calvarial osteoblasts, to stimulate the differentiation of osteoblasts, and to increase the transcript levels of osteogenic genes involved in differentiation and mineralization [38]. Yadav and colleagues reported that modifying the two hydroxyl groups into alkoxy groups could lead to synthetic daidzein derivatives with altered potency [39]. One such compound, 7-(2-diethylamino-ethoxy)-3-(4-methoxyphenyl)-4H-chromen-4-one, increased mineralization of bone marrow osteoprogenitor cells and increased mRNA expression of bone morphogenetic protein-2 and osteocalcin [39]. Our approach only modified the 7-hydroxy moiety by substituting the hydrogen with an isopropyl (daidzein analog 2c), a cyclopentyl (daidzein analog $2 \mathrm{~g}$ ), or an allyl (daidzein analog 2l) while retaining the 4-hydroxy moiety, rather than modifying both hydroxyl groups. We have previously studied the effect of such structural modifications on the estrogenic activity of daidzein analogs and demonstrated the sensitivity of 7-hydroxy substitution to the agonist/antagonist propensity of the daidzein derivatives [21]. While all three analogs have lower estrogenic activity than daidzein [21,22], the specific alkyl substitution of the 7-hydroxy hydrogen yielded significantly increased osteogenic activity. Higher dosages of compounds $2 \mathrm{~g}$ and $2 \mathrm{l}$ in our study did not negatively impact the osteogenic activity of the cells, nor lead to cytotoxicity. Additional studies of structure-activity relationships are underway in our laboratories to determine whether further structural alterations at the other sites will provide increased potency and/or maintain the enhanced efficacy that has been gained by modifications of the 7 hydroxy moiety.

Furthermore, previous studies have also attributed the osteogenic effects of daidzein to the production of equol in the gut. Our previous study thus focused on structurally modifying daidzein to generate equol analogs and investigated whether the equol structural motif conferred greater osteogenic potency [20]. However, our results suggested that the daidzein analogs investigated in this paper, $2 \mathrm{~g}$ and 2l, demonstrated greater osteogenic activity than the equol analogs. Additional equol analogs are being prepared in our laboratory, which may provide more definitive evidence for enhance osteogenic activity compared with the equol analogs investigated previously.

Although analog $2 \mathrm{~g}$ possessed much weaker estrogenic activity than E2, $2 \mathrm{~g}$ treatment enhanced RUNX2, SPARC, and IGF1 in BMSCs and enhanced RUNX2 and COL1A1 in ASCs. RUNX2 is essential for osteoblast development and proper bone formation, regulating transcription of numerous genes that control osteoblast development from mesenchymal stem cells and maturation [40,41]. SPARC, IGF1, and COL1A1 have all been implicated in increasing $\mathrm{BMD}$, increasing biomechanical strength, and maintaining the extracellular matrix [42]. The inhibitory effect of fulvestrant on the osteogenic activity of analog $2 \mathrm{~g}$-treated BMSCs and ASCs provide additional support for the estrogenic activity of $2 \mathrm{~g}$, suggesting that the weaker analog $2 \mathrm{~g}$ appears to enhance osteogenic activity through signaling pathways associated with ER.

Likewise, structural modification of daidzein into ana$\log 2 \mathrm{l}$ also enhanced osteogenic differentiation of BMSCs and ASCs, possibly through ER pathways. Interestingly, unlike analog $2 \mathrm{~g}$, fulvestrant treatment did not abolish the osteogenic activity of analog $2 \mathrm{l}$, suggesting that $2 \mathrm{l}$ is likely to be acting through an ER-independent mechanism. To confirm this finding, the downstream effect of analog $2 \mathrm{l}$ on BMSCs and ASCs were explored in this study. The molecule targeted by analog 21 will probably converge with the identification of key downstream osteogenic genes identified here. BMSCs and ASCs treated with analog 21 demonstrated alterations in the transcriptional level of select early, middle, and late osteogenic genes involved in differentiation of the cells and mineralization of the extracellular matrix. In BMSCs treated with analog 21, all osteogenic genes were upregulated, suggesting a powerful effect of 21 on BMSCs. In contrast, ASCs treated with analog 21 demonstrated a less pronounced effect, altering only c-FOS, COL1A1, and IGF1 [43].

While our study focused on osteogenic activity of the daidzein analogs, it should be noted that the daidzein analogs reduced adipogenic differentiation at $1 \mu \mathrm{M}$ concentration. While our treatment of genistein and daidzein did not yield significant reduction in BMSCs and ASC adipogenic differentiation, it is possible that the dosage used was not adequate to see an effect. Kim and colleagues determined that a $20 \mu \mathrm{M}$ concentration was necessary to reduce adipogenic differentiation of ASCs, and higher concentrations resulted in greater inhibition [44]. Delivery of daidzein also reduced body weight in obesity-induced animals and reduced the expression of adipogenic genes in a dose-dependent manner [44-46]. 
Additional studies have shown that daidzein derivatives have likewise been effective in treating high-fat dietinduced male obese mice [47]. These previous studies, in conjunction with our current study, would suggest further investigating the use of daidzein analogs to reduce diet-induced obesity, as these analogs were more potent than daidzein.

To our knowledge, this study is the first to examine the effects of genistein or daidzein on bone differentiation in human ASCs. Our study demonstrated that these compounds have similar effects on ASCs as on BMSCs. Our study of daidzein analogs further shows that minor structural modifications of daidzein further increased the osteogenic differentiation of BMSCs and ASCs, although the effect was more pronounced in ASCs. Both BMSCs and ASCs are derived from the same germ layer and as such possess similar biologic characteristics. However, indepth analysis of these two cell types has recently revealed differences in immunophenotypical and gene expression profiles [48-50]. Monaco and colleagues determined that ASCs have larger lipid metabolism, migration, and immunomodulatory capacity during early osteogenic differentiation compared with BMSCs, while BMSCs have larger induction of inflammation, cell growth, and proliferation [48]. Consistent with previous reports, our study suggests that differences exist between BMSCs and ASCs which may account for the differences in the transcript levels of osteogenic genes following induction by daidzein analogs. More specifically, BMSCs and ASCs treated with the same daidzein analog express different mRNA levels of osteogenic factors. Furthermore, recent studies have combined the availability of ASCs and their osteogenic differentiation for tissue engineering [51]. ASCs and BMSCs have been seeded onto extracellular matrix scaffolds to increase bone formation [52]. The daidzein analogs investigated in this study could be coated onto these scaffolds to determine their in vivo efficacy in future experiments.

Lastly, as these compounds would be used in postmenopausal women, it is important for these compounds to have no effect on breast cancer and endometrial cancer. Theoretically, a drug used to treat osteoporosis should increase BMD without increasing the risk of cancer. An important advantage of the daidzein analogs compounds is the limited estrogenic effect of these compounds on cancer cells. Previous studies have determined that daidzein and daidzein derivatives have negligible effects on cancer growth or progression [21,53-57]. As such, it is possible that these compounds could be used in combination with chemotherapy or other forms of cancer therapy to reduce the incidence of osteoporosis in at-risk patients. Furthermore, these compounds could be used in combination with other anti-osteoporotic drugs to reduce osteoporosis [58]. However, additional in vivo analyses of these compounds in osteoporosis-induced ovariectomized mice or rats or in critical-sized calvarial defect models are necessary to determine the regenerative potential of these compounds as well as to determine the pharmacokinetics of these compounds for potential combination therapy.

\section{Conclusion}

Osteoporosis is a debilitating disease associated with reduced BMD, increased incidence of fractures, poor mobility, and increased morbidity and mortality. While current therapeutic interventions have been focused on anti-resorptive drugs, development of anabolic drugs that increase bone regeneration is necessary to compensate for bone loss during osteoporosis. In this study, the use of synthetic daidzein derivatives was investigated for the induction of in vitro osteogenesis in BMSCs and ASCs and provided potential mechanisms of action of these compounds. While these data provide a foundation for further analyses of the daidzein analogs in BMSCs or ASCs, in vitro or in vivo, future studies are necessary to investigate the role of these compounds in a rodent ovariectomized model of osteoporosis on BMD and bone architecture and for the translation of these compounds clinically to improve the outcomes of patients diagnosed with osteoporosis.

\section{Additional files}

\begin{abstract}
Additional file 1: Shows a characterization of BMSCs and ASCs.
BMSCs $(n=6)$ and ASCs $(n=6)$ were each induced to undergo

osteogenic and adipogenic differentiation, immunophenotyped for cell surface antigens with flow cytometry, and the colony formation potential determined. (A) BMSCs and ASCs were cultured in ODM or ADM for 14 days and stained with alizarin red or oil red $O$, respectively. Representative images of osteogenesis ( $4 \times$ magnification) and adipogenesis (10X magnification) in BMSCs and ASCs are shown. Scale bar represents $200 \mu \mathrm{m}$. (B) Cells were stained with cell surface markers, CD44, CD90, CD106, CD166, CD11b, CD34, and CD45 and their respective isotype controls. Each overlay contains the isotype control for each cell type and the cell surface marker of interest. (C) BMSCs and ASCs were plated at 100 cells per $10 \mathrm{~cm}$ plate and were stained with crystal violet after 14 days in culture to visualize the colony-forming units.

Additional file 2: Shows daidzein analogs inhibit adipogenesis. BMSCs $(n=6)$ and ASCs $(n=6)$ were each induced to undergo adipogenic differentiation and treated with vehicle, E2, daidzein, or daidzein analog $(1 \mu \mathrm{M})$ for 14 days and stained with oil red $\mathrm{O}$. (A) Representative images of cells stained with oil red $\mathrm{O}$ are shown at 10X magnification. Scale bar represents $100 \mu \mathrm{m}$. (B) To quantify the amount of oil red $\mathrm{O}$ staining in treated BMSCs and ASCs, cells were eluted with isopropanol and measured at $544 \mathrm{~nm}$. Adipogenic differentiation was determined relative to vehicle-treated cells (normalized to 1.0). Bars, \pm standard deviation. ${ }^{*} P<0.05$; ${ }^{* *} P<0.01$; ${ }^{* *} P<0.001$.
\end{abstract}

Additional file 3: Shows analogs $2 \mathrm{~g}$ and $2 \mathrm{l}$ do not demonstrate cytotoxic or proliferative effects on BMSCs and ASCs. (A) BMSCs $(n=6)$ and (B) ASCs $(n=6)$ were each cultured in CCM supplemented with vehicle or daidzein analog $(1 \mu \mathrm{M})$ for 7 or 14 days and assessed by MTT assay. Bars, \pm standard deviation.

Additional file 4: Presents the $\mathrm{EC}_{50}$ of each compound in BMSCs and ASCs. Values presented in molar (M) concentration.

Additional file 5: Presents the gene expression profile of BMSCs and ASCs treated with E2, daidzein, analog $2 \mathrm{~g}$, analog or $2 \mathrm{l}$ in the presence and absence of fulvestrant on day 3. Data normalized to 
vehicle-treated cells after 3 days. ${ }^{*} P<0.05 ;{ }^{* *} P<0.01 ;{ }^{* * *} P<0.001$ relative to the respective vehicle-treated cells.

Additional file 6: Shows that ER antagonist fulvestrant inhibits the expression of osteogenic genes induced by daidzein analogs in BMSCs. BMSCs were cultured in CDS-ODM and concurrently treated with vehicle, E2 $(10 \mathrm{nM})$, daidzein $(1 \mu \mathrm{M})$, or daidzein analog $(1 \mu \mathrm{M})$ and ER antagonist fulvestrant. Cells were collected after 3, 7, or 14 days of treatment. RNA was isolated from the cells and reverse transcribed into cDNA. Analyses of osteogenic genes were assessed by quantitative polymerase chain reaction. Expression values are normalized to undifferentiated cells, normalized to 1.0. Bars, \pm standard deviation

Additional file 7: Presents the gene expression profile of BMSCs and ASCs treated with E2, daidzein, analog $2 \mathrm{~g}$, or analog $2 \mathrm{l}$ in the presence and absence of fulvestrant on day 7. Data normalized to vehicle-treated cells after 7 days. ${ }^{*} P<0.05 ;{ }^{* *} P<0.01 ;{ }^{* * *} P<0.001$ relative to the respective vehicle-treated cells.

Additional file 8: Presents the gene expression profile of BMSCs and ASCs treated with E2, daidzein, analog $2 \mathrm{~g}$, or analog $2 \mathrm{l}$ in the presence and absence of fulvestrant on day 14 . Data normalized to vehicle-treated cells after 14 days. ${ }^{*} P<0.05$; ${ }^{* *} P<0.01$; ${ }^{* * *} P<0.001$ relative to the respective vehicle-treated cells.

Additional file 9: Shows that fulvestrant-treated ASCs demonstrate reduced expression of osteogenic transcription factors induced by daidzein analogs. ASCs were cultured in CDS-ODM and concurrently treated with vehicle, E2 $(10 \mathrm{nM})$, daidzein $(1 \mu \mathrm{M})$, or daidzein analog $(1 \mu \mathrm{M})$ and ER antagonist fulvestrant $(100 \mathrm{nM})$. Cells were collected after 3,7 , or 14 days of treatment. RNA was isolated from the cells and reverse transcribed into CDNA. Analyses of osteogenic genes were assessed by quantitative polymerase chain reaction. Expression values are normalized to undifferentiated vehicle-treated cells, normalized to 1.0. Bars, \pm standard deviation. ${ }^{*} P<0.05 ;{ }^{*} P<0.01 ;{ }^{* * P} P 0.001$ relative to vehicle-treated cells.

Additional file 10: Presents the gene expression profile of BMSCs and ASCs treated with analog $2 \mathrm{l}$ in the presence and absence of fulvestrant on day 14. Data normalized to vehicle-treated cells after 14 days. ${ }^{*} P<0.05$; relative to the respective vehicle-treated cells.

\section{Abbreviations}

ALP: alkaline phosphatase; ASC: adipose-derived stromal/stem cell; BCIP/ NBT: 5-bromo-4-chloro-3-indolyl phosphate; BMD: bone mineral density; BMSC: bone marrow-derived mesenchymal stem cell; CCM: complete culture media; CDS-ADM: charcoal dextran-stripped adipogenic differentiation medium; CDS-ODM: charcoal dextran-stripped osteogenic differentiation medium; c-FOS: FBJ murine osteosarcoma viral oncogene homolog: $\mathrm{CO}_{2}$ : carbon dioxide; COL1A1: collagen type 1 alpha; DLX5: distal-less homeobox 5: DMP1: dentin matrix acidic phosphoprotein 1: E2: 17ßestradiol; $\mathrm{EC}_{50}$ : half-maximal effective concentration; EDTA: ethylenediamine tetraacetic acid; ER: estrogen receptor; IGF1: insulin-like growth factor 1; ODM: osteogenic differentiation medium; PBS: phosphate-buffered saline; RUNX2: runt-related transcription factor 2; SOST: sclerostin; SPARC: osteonectin; SPP1: secreted phosphoprotein 1.

\section{Competing interests}

JMG is the co-founder, co-owner, and Chief Scientific Officer of LaCell LLC, a for profit biotech company focusing on the use of of stromal/stem cells. The remaining authors declare that they have no competing interests.

\section{Authors' contributions}

ALS conceived the study, participated in its design and coordination, collected, analyzed and interpreted the data, performed statistical analysis, and manuscript writing. JFO and SE carried out the experiments. QJ, QZ, SZ SMB, and GW synthesized and prepared the daidzein analogs. JMG helped analyze and interpret the data. MEB, GW, and BAB conceived the study, participated in the design of the study, interpreted the data, and provided financial support. GW designed the daidzein analogs. BAB also participated in manuscript writing. All authors read and approved the final manuscript.

\section{Acknowledgements}

This study was supported by the National Center for Research Resources RCMI program through Grant 1G12RR026260-01, US Department of Agriculture Grant 58-6435-7-019, the Louisiana Cancer Research Consortium, National Institute of General Medical Sciences of the National Institutes of Health 1 U54GM104940 (Louisiana Clinical and Translational Science Center), and funds from Tulane University pilot grant

\section{Author details}

Center for Stem Cell Research and Regenerative Medicine, Tulane University School of Medicine, 1430 Tulane Avenue, SL-99, New Orleans, LA 70112, USA. ${ }^{2}$ Department of Chemistry and RCMI Cancer Research Program, Xavier University of Louisiana, New Orleans, LA 70125, USA. ${ }^{3}$ Southern Regional Research Center, US Department of Agriculture, 1100 Robert E. Lee Blvd, New Orleans, LA 70124, USA. ${ }^{4}$ Department of Medicine, Tulane University School of Medicine, New Orleans, LA 70112, USA.

Received: 7 February 2014 Revised: 5 August 2014

Accepted: 6 August 2014 Published: 28 August 2014

\section{References}

1. Bliuc D, Nguyen ND, Milch VE, Nguyen TV, Eisman JA, Center JR: Mortality risk associated with low-trauma osteoporotic fracture and subsequent fracture in men and women. JAMA 2009, 301:513-521.

2. Johnell O, Kanis JA, Oden A, Sernbo I, Redlund-Johnell I, Petterson C, De Laet C, Jonsson B: Mortality after osteoporotic fractures. Osteoporis Int 2004, 15:38-42.

3. Center JR, Nguyen TV, Schneider D, Sambrook PN, Eisman JA: Mortality after all major types of osteoporotic fracture in men and women: an observational study. Lancet 1999, 353:878-882.

4. Weiner JM, Tilly J: Population ageing in the United States of America: implications for public programmes. Int J Epidemiol 2002, 31:776-781.

5. Burge R, Dawson-Hughes B, Solomon DH, Wong JB, King A, Tosteson A: Incidence and economic burden of osteoporosis-releated fractures in the United States, 2005-2025. J Bone Miner Res 2007, 22:465-475.

6. Smith EL, Raab DM: Osteoporosis and physical activity. Acta Med Scand 2009, 220:149-156.

7. Beard MK: Bisphosphonate therapy for osteoporosis: combining optimal fracture risk reduction with patient preference. Curr Med Res Opin 2012, 28:141-147.

8. Uihlein AV, Leder BZ: Anabolic therapies for osteoporosis. Endocrinol Metab Clin North Am 2012, 41:507-525.

9. Khastgir G, Studd J, Holland N, Alaghband-Zadeh J, Fox S, Chow J: Anabolic effect of estrogen replacement on bone in postmenopausal women with osteoporosis: histomorphometric evidence in a longitudinal study. J Clin Endocrinol Metab 2001, 86:289-295.

10. Okazaki R, Inoue D, Shibata M, Saika M, Kido S, Ooka H, Tomiyama H, Sakamoto Y, Matsumoto T: Estrogen promotes early osteoblast differentiation and inhibits adipocyte differentiation in mouse bone marrow stromal cell lines that express estrogen receptor (ER) alpha or beta. Endocrinology 2002, 143:2349-2356.

11. Collaborative Group on Hormonal Factors in Breast Cancer: Breast cancer and hormone replacement therapy: collaborative reanalysis of data from 51 epidemiological studies of 52,705 women with breast cancer and 108,411 women without breast cancer. Collaborative Group on Hormonal Factors in Breast Cancer. Lancet 1997, 350:1047-1059.

12. Schairer C, Lubin J, Troisi R, Sturgeon S, Brinton L, Hoover R: Menopausal estrogen and estrogen-progestin replacement therapy and breast cancer risk. JAMA 2000, 283:485-491.

13. Colditz GA, Hankinson SE, Hunter DJ, Willett WC, Manson JE, Stampfer MJ, Hennekens C, Rosner B, Speizer FE: The Use of estrogens and progestins and the risk of breast cancer in postmenopausal women. N Engl J Med 1995, 332:1589-1593.

14. Ettinger B, Black DM, Mitlak BH, Knickerbocker RK, Nickelsen T, Genant HK, Christiansen C, Delmas PD, Zanchetta JR, Stakkestad J, Gluer CC, Krueger K, Cohen FJ, Eckert S, Ensrud KE, Avioli LV, Lips P, Cummings SR: Reduction of vertebral fracture risk in postmenopausal women with osteoporosis treated with raloxifene: results from a 3-year randomized clinical trial. JAMA 1999, 282:637-645. 
15. Mosca L, Grady D, Barrett-Connor E, Collins P, Wenger N, Abramson BL, Paganini-Hill A, Geiger MJ, Dowsett SA, Amewou-Atisso M, Kornitzer M: Effect of raloxifene on stroke and venous thromboembolism according to subgroups in postmenopausal women at increased risk of coronary heart disease. Stroke 2009, 40:147-155.

16. Tuku K, Melby MK, Kurzer MS, Mizuno S, Watanabe S, Ishimi Y: Effects of soy isoflavone supplements on bone turnover markers in menopausal women: systematic review and meta-analysis of randomized controlled trials. Bone 2010, 47:413-423

17. Alekel DL, Van Loan MD, Koehler KJ, Hanson LN, Stewart JW, Hanson KB, Kurzer MS, Peterson CT: The Soy Isoflavones for Reducing Bone Loss (SIRBL) Study: a 3-y randomized controlled trial in postmenopausal women. Am J Clin Nutr 2010, 91:218-230.

18. Bhargavan B, Gautam AK, Singh D, Kumar A, Chaurasia S, Tyagi AM, Yadav DK, Mishra JS, Singh AB, Sanyal S, Goel A, Maurya R, Chattopadhyay N: Methoxylated isoflavones, cajanin and isoformononetin, have non-estrogenic bone forming effect via differential mitogen activated protein kinase (MAPK) signaling. J Cell Biochem 2009, 108:388-399.

19. de Wilde A, Lieberherr M, Colin C, Pointillart A: A low dose of daidzein acts as an ERß-selective agonist in trabecular osteoblasts of young female piglets. J Cell Physiol 2004, 200:253-262.

20. Vitale DC, Piazza C, Melilli B, Drago F, Salomone S: Isoflavones: estrogenic activity, biological effect and bioavailability. Eur J Drug Metab Pharmacokinet 2013, 38:15-25.

21. Jiang Q, Payton-Stewart F, Elliott S, Driver J, Rhodes LV, Zhang Q, Zheng S, Bhatnagar D, Boue SM, Collins-Burow BM, Sridhar J, Stevens C, McLachlan JA, Wiese TE, Burow ME, Wang G: Effects of 7-O substitutions on estrogenic and anti-estrogenic activities of daidzein analogues in MCF-7 breast cancer cells. J Med Chem 2010, 53:6153-6163.

22. Strong AL, Jiang $Q$, Zhang $Q$, Zheng S, Bou SM, Elliott S, Burow ME, Bunnell BA, Wang G: Design, synthesis, and osteogenic activity of daidzein analogs on human mesenchymal stem cells. ACS Med Chem Lett 2013, 5:143-148.

23. Jakob F, Ebert $R$, Ignatius $A$, Matsushita $T$, Watanabe $Y$, Groll J, Walles $H$ : Bone tissue engineering in osteoporosis. Maturitas 2013, 75:118-124.

24. Gimble JM, Katz AJ, Bunnell BA: Adipose-derived stem cells for regenerative medicine. Circ Res 2007, 100:1249-1260.

25. Faulds MH, Zhao C, Dahlman-Wright K, Gustafsson J-A: The diversity of sex steroid action: regulation of metabolism by estrogen signaling. J Endocrinol 2012, 212:3-12.

26. Filardo EJ, Thomas P: Minireview: G protein-coupled estrogen receptor-1, GPER-1: its mechanism of action and role in female reproductive cancer, renal and vascular physiology. J Endocrinol 2012, 153:2953-2962

27. Prossnitz $E R$, Barton M: Estrogen biology: new insights into GPER function and clinical opportunities. Mol Cell Endocrinol 2014, 389:71-83.

28. McDonnell DP, Wardell SE: The molecular mechanisms underlying the pharmacological actions of ER modulators: implications for new drug discovery in breast cancer. Curr Opin Pharmacol 2010, 10:620-628.

29. Wang MW, Traystman RJ, Hurn PD, Liu T: Non-classical regulation of estrogen receptor-alpha by ICl182,780. J Steroid Biochem Mol Biol 2004, 92:51-62.

30. Wardell SE, Kazmin D, McDonnell DP: Research Resource: transcriptional profiling in a cellular model of breast cancer reveals functional and mechanistic differences between clinically relevant SERM and between SERM/Estrogen complexes. Mol Endocrinol 2012, 26:1235-1248.

31. Bitto A, Burnett BP, Polito F, Marini H, Levy RM, Armbruster MA, Minutoli L, Stefano VD, Irrera N, Antoci S, Granese R, Squadrito F, Altavilla D: Effects of genistein aglycone in osteoporotic, ovariectomized rats: a comparison with alendronate, raloxifene and oestradiol. Br J Pharmacol 2008, 155:896-905.

32. Bitto A, Polito F, Squadrito F, Marini H, D'Anna R, Irrera N, Minutoli L, Granese R, Altavilla D: Genistein aglycone: a dual mode of action anti-osteoporotic soy isoflavone rebalancing bone turnover towards bone formation. Curr Med Chem 2010, 17:3007-3018.

33. Picherit C, Coxam V, Bennetau-Pelissero C, Kati-Coulibaly S, Davicco M-J, Lebecque $\mathrm{P}$, Barlet J-P: Daidzein is more efficient than genistein in preventing ovariectomy-induced bone loss in rats. J Nutr 2000, 130:1675-1681.

34. Wong RWK, Rabie ABM: Effect of daidzein on bone formation. Front Biosci $2009,14: 3673-3679$
35. Rassi CM, Lieberherr M, Chaumaz G, Pointillart A, Cournot G: Down-regulation of osteoclast differentiation by daidzein via caspase 3. J Bone Miner Res 2002, 17:630-638.

36. Fonseca D, Ward WE: Daidzein together with high calcium preserve bone mass and biomechanical strength at multiple sites in ovariectomized mice. Bone 2004, 35:489-497.

37. Wang SF, Jiang Q, Ye YH, Li Y, Tan RX: Genistein derivatives as selective estrogen receptor modulators: sonochemical synthesis and in vivo anti-osteoporotic action. Bioorg Med Chem Lett 2005, 13:4880-4890.

38. Kumar M, Rawat P, Kureel J, Singh AK, Singh D, Maurya R: One step synthesis of 2-hydroxymethylisoflavone and their osteogenic activity. Bioorg Med Chem Lett 2011, 21:1706-1709.

39. Yadav DK, Gautam AK, Kureel J, Srivastava K, Sahai M, Singh D, Chattopadhyay N, Maurya R: Synthetic analogs of daidzein, having more potent osteoblast stimulating effect. Bioorg Med Chem Lett 2011, 21:677-681.

40. Schroeder TM, Jensen ED, Westendorf JJ: Runx2: a master organizer of gene transcription in developing and maturing osteoblasts. Birth Defects Res C Embryo Today 2005, 75:213-225.

41. Augello A, De Bari C: The regulation of differentiation in mesenchymal stem cells. Hum Gene Ther 2010, 21:1226-1238.

42. Delany AM, Amling M, Priemel M, Howe C, Baron R, Canalis E: Osteopenia and decreased bone formation in osteonectin-deficient mice. J Clin Invest 2000, 105:915-923

43. Kalajzic I, Staal A, Yang W-P, Wu Y, Johnson SE, Feyen JHM, Krueger W, Maye P, Yu F, Zhao Y, Kuo L, Gupta RR, Achenie LE, Wang HW, Shin DG, Rowe DW: Expression profile of osteoblast lineage at defined stages of differentiation. J Biol Chem 2005, 280:24618-24626.

44. Kim MH, Park JS, Seo MS, Jung JW, Lee YS, Kang KS: Genistein and daidzein repress adipogenic differentiation of human adipose tissue-derived mesenchymal stem cells via $W n t / \beta$-catenin signalling or lipolysis. Cell Prolif 2010, 43:594-605.

45. Crespillo A, Alonso M, Vida M, Pavón F, Serrano A, Rivera P, Romero-Zerbo Y, Fernández-Llebrez P, Martínez A, Pérez-Valero V, Bermúdez-Silva FJ, Suárez J, de Fonseca FR: Reduction of body weight, liver steatosis and expression of stearoyl-CoA desaturase 1 by the isoflavone daidzein in diet-induced obesity. Br J Pharmacol 2011, 164:1899-1915.

46. Kim MH, Park H, Kim WG, Lee YS: The inhibitory effects of soy daidzein on obesity in C57BI/6J mice fed high fat A1099. FASEB J 2007, 21:851-858.

47. Guo Y, Wu G, Yang H, Zhang J: Antiobesity action of a daidzein derivative on male obese mice induced by a high-fat diet. Nutr Res 2009, 29:656-663.

48. Monaco E, Bionaz M, Rodriguez-Zas S, Hurley WL, Wheeler MB: Transcriptomics comparison between porcine adipose and bone marrow mesenchymal stem cells during in vitro osteogenic and adipogenic differentiation. PLOS One 2012, 7:1-19.

49. Pachon-Pena G, Yu G, Tucker HA, Wu X, Vendrell J, Bunnell BA, Gimble JM: Stromal stem cells from adipose tissue and bone marrow of agematched female donors display distinct immunophenotypic profiles. J Cell Physiol 2011, 226:843-851.

50. Strioga M, Viswanathan S, Darinskas A, Slaby O, Michalek J: Same or not the same? Comparison of adipose tissue-derived versus bone marrow-derived mesenchymal stem and stromal cells. Stem Cells Dev 2012, 21:2724-2752

51. Rada T, Reis RL, Gomes ME: Adipose tissue-derived stem cells and their application in bone and cartilage tissue engineering. Tissue Eng Part B Rev 2009, 15:113-125.

52. Guven S, Mehrkens A, Saxer F, Schaefer DJ, Martinetti R, Martin I, Scherberich A: Engineering of large osteogenic grafts with rapid engraftment capacity using mesenchymal and endothelial progenitors from human adipose tissue. Biomaterials 2011, 32:5801-5809

53. Gercel-Taylor C, Feitelson AK, Taylor DD: Inhibitory effect of genistein and daidzein on ovarian cancer cell growth. Anticancer Res 2004, 24:795-800.

54. Jin S, Zhang QY, Kang XM, Wang JX, Zhao WH: Daidzein induces MCF-7 breast cancer cell apoptosis via the mitochondrial pathway. Ann Oncol 2010, 21:263-268.

55. Ju YH, Fultz J, Allred KF, Doerge DR, Helferich WG: Effects of dietary daidzein and its metabolite, equol, at physiological concentrations on 
the growth of estrogen-dependent human breast cancer (MCF-7) tumors implanted in ovariectomized athymic mice. Carcinogenesis 2006 27:856-863.

56. Lamartiniere CA, Wang J, Smith-Johnson M, Eltoum I-E: Daidzein: bioavailability, potential for reproductive toxicity, and breast cancer chemoprevention in female rats. Toxicol Sci 2002, 65:228-238.

57. Liu X, Suzuki N, Laxmi YRS, Okamoto Y, Shibutani S: Anti-breast cancer potential of daidzein in rodents. Life Sci 2012, 91:415-419.

58. Park $C Y$, Weaver $C M$ : Vitamin $D$ interactions with soy isoflavones on bone after menopause: a review. Nutrients 2012, 4:1610-1621.

doi:10.1186/scrt493

Cite this article as: Strong et al:: Novel daidzein analogs enhance osteogenic activity of bone marrow-derived mesenchymal stem cells and adipose-derived stromal/stem cells through estrogen receptor dependent and independent mechanisms. Stem Cell Research \& Therapy 2014 5:105

\section{Submit your next manuscript to BioMed Central} and take full advantage of:

- Convenient online submission

- Thorough peer review

- No space constraints or color figure charges

- Immediate publication on acceptance

- Inclusion in PubMed, CAS, Scopus and Google Scholar

- Research which is freely available for redistribution 